\title{
Discursos silenciados: poder y interdicción en los registros epistolares del sanatorio sonnenfelds durante la segunda guerra mundial*
}

\author{
Silenced speeches: power and interdiction in the epistolary \\ records of the sonnenfelds sanitarium during the second world
}

PP. 9-20

Marcio de Lima Pacheco**

REC: $14 / 05 / 2020$

Plinio Pereira Filho ${ }^{\star \star \star}$

ACEP: $31 / 08 / 2020$

\begin{abstract}
Resumen
La historia de los sanatorios en la Segunda Guerra Mundial en Europa no terminó con la caída del Sonnenfelds en Alemania en 2012. Todavía hay mucho que decir al respecto. El presente artículo tiene el objetivo de traer a colación discusiones al analizar la historia y el proceso de producción y circulación de las últimas e inéditas cartas en un sanatorio en
\end{abstract}

la Alemania nazi. ¿Qué práctica discursiva se muestra en las cartas a partir de los mecanismos de interdicción del discurso? La investigación se en los presupuestos teóricos del análisis de discurso (AD) de línea francesa, sobre todo dentro de los postulados de Michel Foucault sobre el discurso, nacimiento de la clínica, historia de la locura y mecanismos

\footnotetext{
* Ese articulo es una investigación hecha sobre las inéditas y únicas cartas que quedaran del Sanatorio Sonnenfelds durante la segunda Grand Guerra y se desarolló como pesquisa de Doctorado y PosDoctorado en la Universidad del Estado de Rio Grande do Norte. Ese estudio científico está vinculado al Grupo de Teoria Política Contemporânea del Departamiento de Filosofia de la Universidad Federal de Rondônia (UNIR).

** Licenciado en Filosofia por la Universidad del Estado do Rio Grande do Norte (UERN). Bacharel en Teologia por la Facultad Católica Dehoniana. Maestría en Filosofía/Metafísica de la Universidad Federal do Rio Grande do Norte(UFRN). Doctorado en Filosofía/Metafísica de la Pontifícia Universidad Católica de São Paulo(PUC-SP) y Posdoctorado en Letras, Lingüística y Discurso de la Universidad do Estado do Rio Grande do Norte (UERN). Chefe, Coordenador y Docente do Departamiento de Filosofia da Universidade Federal de Rondônia (UNIR).Evaluador del Instituto Nacional de Estudos e Pesquisas Educacionais/Ministério da Educação (INEP/MEC) para los cursos de Filosofía y Teología. Participa del Grupo de Teoria Política Contemporânea vinculado al Departamento de Filosofia da UNIR. E-mail: professor.pacheco@unir.bro ppachecus@hotmail.com; Orcid: http://orcid.org/0000-0003-3902-2680

*** Licenciado en Lenguaje (Lengua Vernácula y Lengua Inglesa) de la Universidad Federal de Campina Grande con Especialización en Psicología Educacional y de Aprendizaje de la Facultad Integrada de Patos, Maestría en Lenguaje de la Universidad Federal de la Paraíbay candidato a doctor en Lenguaje del Programa de Posgrado en Lenguaje de la Universidad del Estado del Rio Grande del Norte y Universidad de São Paulo. Actualmente desarrolla su investigación de Doctorado en Lecturas en las áreas de Análisis del Discurso con énfasis en la historia de las cartas y documentos que circularon en los campos de concentración durante la Segunda Guerra Mundial. Es pesquisador de linguage por la UERN/USP, así como miembro del Grupo de Estudios del Discurso (GRED) de la Universidad de Estado del Rio Grande del Norte, Brasil. Fue docente invitado por la Facultad de Ciencia y Tecnología Docente Dirson Maciel de Barros en Goiana, Pernambuco. Labora como tradutor e pesquisador en la Universidad do Estado do Rio Grande do Norte (UERN) Correo electrónico: professor_plinio@hotmail.com; Orcid: https:// orcid.org/0000-0002-4398-2329
} 
de control. También recurre a la mirada de Orlandi sobre las formas del silencio y sus significaciones; a las contribuciones de Hannah Arendt sobre las facetas del antisemitismo, el Estado totalitario Nazi, la ideología y el terror como forma de gobierno. La metodología se basa en el método cualitativo y buscó analizar la carta intercambiada entre la pareja Ulli y Rudi durante el período de la Segunda Gran Guerra. A través del recorrido histórico con respecto a las manifestaciones del poder del régimen de control y prohibición, se percibe en las análisis de fragmentos en las cartas producidas dentro del sanatorio que los sujetos internados eran sometidos al control, también a la producción discursiva y en la mayoría de las veces, no tenían acceso a información que viniera de afuera.

Palabras clave: Sanatorio de Sonnenfelds, Análisis del Discurso, práctica discursiva, control y prohibición

\section{Abstract}

The history of sanatoriums in World War II in Europe did not end with the fall of the Sonnenfelds in Germany in 2012. There is still much to be said about it. This article aims to bring up discussions by analyzing the history and the process of production and circulation of the latest and unpublished letters in a sanitarium in Nazi Germany. What discursive practice is shown in the letters from the mechanisms of discourse interdiction? The research is on the theoretical assumptions of French-line discourse analysis (AD), especially within the postulates of Michel Foucault on discourse, the birth of the clinic, the history of madness and control mechanisms. He also draws on Orlandi's gaze on the forms of silence and their meanings; to Hannah Arendt's contributions on the facets of anti-Semitism, the Nazi totalitarian state, ideology and terror as a form of government. The methodology is based on the qualitative method and sought to analyze the letter exchanged between the couple Ulli and Rudi during the period of the Second Great War. Through the historical journey with respect to the manifestations of the power of the control and prohibition regime, it is perceived in the analysis of fragments in the letters produced within the sanatorium that the interned subjects were subjected to control, also to discursive production and in the Most of the time, they did not have access to information that came from outside.

Keywords: Sanatorio of Sonnefields, Discourse Analysis, discursive practice, control and prohibition

\section{Consideraciones iniciales}

De las memorias del nacimiento del Sanatorio Sonnenfelds:

En octubre de 1900 fue oficialmente licenciado el Sanatorio Waldhaus de la hermana Elisabeth Teichgräber en Sülzhayn, que ya había abierto en 1899 como una casa de recreo. Los sanatorios operativos de la hermana Elisabeth Teichgräber, se dividieron inicialmente en dos edificios. Además del sanatorio real, que es conocido por los antiguos habitantes de Sülzhayn como la casa principal, la propiedad en la calle Dr. Kremser, de 74 de hoy, también llamada apéndice del sanatorio Waldhaus ${ }^{1}$ (SÜLZAHYN, 2017, p 01).Traducción propia.

El presente artículo busca interpretar por medio del discurso epistolar de un sanatorio, marcas del silenciamiento y del poder disciplinario en lo que se refiere a la correspondencia durante el III Reich.

La aparición de la clínica como hecho histórico se debe identificar con el sistema de estas

1 “Im Oktober 1900 wurde das Sanatorium 'Waldhaus' der Schwester Elisabeth Teichgräber (1861-1937) in Sülzhayn staatlich konzessioniert, welches bereits 1899 als Erholungshaus eröffnet hatte. Der Heilstättenbetrieb der Schwester Elisabeth Teichgräber verteilte sich zunächst auf zwei Gebäude. Neben dem eigentlichen Sanatorium, das von den älteren Einwohnern Sülzhayns als ‘Haupthaus’ bezeichnet wird, gehörte auch das Anwesen in der heutigen Dr.-Kremser- Straße74 als sogenannter “Appendix’ zum Sanatorium “Waldhaus”. 
reorganizaciones. Esta nueva estructura se revela, pero ciertamente no se agota en el cambio que sustituyó a la pregunta ¿qué es lo que tiene? Desde inicio del siglo XVIII, el diálogo entre el médico y el enfermo, con una gramática y su estilo propio, por otra que se reconoce en el juego de la clínica y el principio de todo discurso: ¿dónde le duele? "La clínica [...] debe su real importancia al hecho de ser una reorganización en profundidad no s[o] lo de los conocimientos médicos, sino de la propia posibilidad de un discurso sobre la enfermedad". (Foucault, 2010 XVIII)

\section{Figura 1.}

Fotografía de la Fundación del sanatorio de Sonnefelds 19.06.1913 - (WACHENFELD. Leonhard, 2017, p.01).

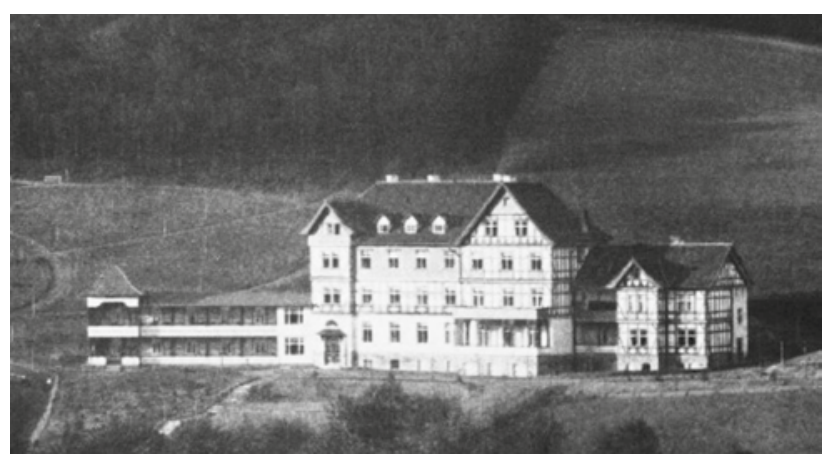

Fuente: http://glass-portal.privat.t-online.de/suelzhayn/architekt/mohr_und_weidner1.2.htm\#source

El 1 을 de mayo de 1912, Wasmund abrió la nueva casa de tratamiento bajo el nombre de Sanatorio Sonnenfields con una capacidad máxima de 35 camas. La construcción costó cerca de 144.000 RM sin casas al lado, fue publicado en 1912 y 1915 en revistas arquitectónicas alemanas. Frieda Wasmund llevó la casa Sonnenfelds hasta 1921 y luego se mudó a Hamburgo.
Figura 2.

Ruinas y abandono lo que en el 2010. (WACHENFELD. Leonhard, 2017, p.01)

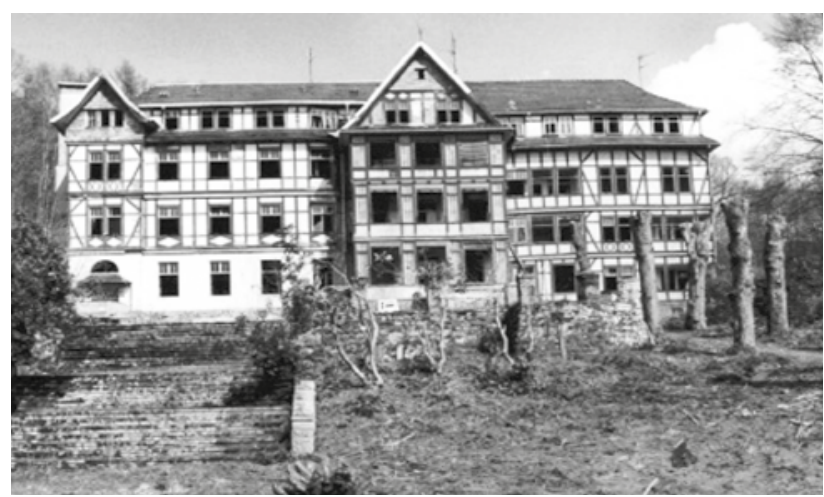

Fuente: http://glass-portal.privat.t-online.de/suelzhayn/architekt/mohr_und_weidner1.2.htm\#source

El sanatorio fue vendido a Karl Reichel. Reichel vivía en Sülzhayn desde febrero de 1913 y fue cofundador con el Dr. Josef Stein y Wilhelm Palm del nuevo sanatorio del Dr. Stein y lo acompañó hasta 1921. Karl Reichel realizó la expansión del Sonnenfelds. El piso superior del sanatorio se amplió. Además del sanatorio, Reichel construyó para sí y su familia una casa en 1929, de acuerdo con los planos del arquitecto Ilfelder Karl. El edificio residencial lateral 1911/12 se aumentó e integró al sanatorio. Después de la liberación del campo de concentración Mittelbar-Dora en Nordhausen / Harz el 11 de abril de 1945, la casa fue ocupada con los prisioneros del antiguo campo de concentración. Después de su regreso a la patria, el sanatorio pasó a la posesión de sitios de casa del VVN G.m.b.H. En 1946, Karl Reichel perdió todos sus bienes por expropiación y fue obligado a vivir en un pequeño apartamento en la casa del Sexton ${ }^{2}$ (vieja escuela) (SÜLZAHYN, 2017, p 1). Traducción propia.

2 Original en Alemán: “Am 1. Mai 1912 eröffnete Frieda Wasmund das neue Kurhaus unter dem Namen ‘Sanatorium Sonnenfels’ mit einer maximalen Kapazität von 35 Betten. Der Bau, der ohne das seitlich angebaute Wohnhaus rund 144.00o RM kostete, wurde 1912 und 1915 in deutschen Architekturzeitschriften publiziert. Frieda Wasmund leitete das Haus 'Sonnenfels' bis 1921 und siedelte dann nach Hamburg über. Das Sanatorium verkaufte sie an Karl Reichel (1879-1963). Reichel lebte bereits seit Februar 1913 in Sülzhayn und war neben Dr. med. Josef Stein (1877-1961) und Wilhelm Palm (1879-1946) Mitbegründer von Dr. Steins Neuem Sanatorium, bis 1921 auch Mitbesitzer. Karl Reichel veranlasste bald die Erweiterung von 'Sonnenfels'. Das Dachgeschoß des Sanatoriums wurde ausgebaut, die Loggia bis ins 2. Obergeschoß erhöht. Neben dem Sanatorium ließ Reichel für sich und seine Familie 1929 ein Wohnhaus nach Plänen des Ilfelder Architekten Karl Picking (1888-1962) errichten. Das 1911/12 seitlich angebaute Wohngebäude wurde aufgestockt und in das Sanatorium integriert. Nach der Befreiung des Konzentrationslagers 'Mittelbau-Dora' bei Nordhausen/Harz am 11. April 1945 wurde das Haus 'Sonnenfels' mit ehemaligen KZ-Häftlingen belegt. Nach deren Rückführung in die Heimat ging das Sanatorium in den Besitz der VVN-Heimstätten G.m.b.H. über. Karl Reichel verlor 1946 durch Enteignung seinen gesamten Besitz und wurde in eine kleine Wohnung im Küsterhaus (Alte Schule). 
El decir en los documentos de la Segunda Gran Guerra es histórico, pues enuncia a partir de un determinado lugar (sanatorios) y en un determinado tiempo (1945) los sentidos que ese sujeto produjo, son determinados por la manera en que él se inscribe en la lengua y en la lengua histórica y no únicamente, por su voluntad. En este sentido, de acuerdo con Orlandi (2007, p. 42),

[...] el sentido no existe en sí, pero es determinado por las posiciones ideológicas colocadas en juego en el proceso socio-histórico en que las palabras son producidas. Las palabras cambian de sentido según las posiciones de aquellos que las emplean.

Así como Ulli y Rudi, muchos otros pueblos vivieron sobre la égida de la dominación y de la ideología del gobierno totalitario nazi en la Segunda Guerra. Los gobiernos totalitarios se fundamentan en la ideología del dominio total a través del terror, lejos de cualquier humanidad y de su racionalidad. Sistematizan la violencia de forma burocrática y deshacen enteramente al hombre de su propia realidad y humanidad. Traen el terror al terreno de la institución de forma administrativa y lógica.

\section{Para Foucault,}

[...] la raza, el racismo, es la condición de aceptación de la vida en una sociedad de normalización. [...] La función asesina del Estado s[o]lo puede ser asegurada, siempre que el Estado funcione en el modo del biopoder, por el racismo. (Foucault, 1975-1976/2005, p.306)

Por lo tanto, según el filósofo, "el racismo está ligado al funcionamiento de un Estado que está obligado a utilizar la raza, la eliminación de las razas y la purificación de la raza para ejercer su poder soberano" (Foucault, 1975-1976/2005, p. 309). Esto, por lo tanto, alcanza su auge con el régimen nazi.
Es con base en las memorias del sujeto que pasó por este régimen de racismo, persecución y miedo en la Segunda Guerra, que se hace un abordaje al poder como fuerza represora del régimen nazi hasta llegar a la producción del discurso y de su respectiva interdicción por medio de cartas. Tangencialmente, se aproxima un poco el silencio como forma de traer significados lo que fue o dejó de ser silenciado en la censura de las cartas. Se ha hablado mucho del régimen nazi como movimiento histórico, pero poco se publica, sobre todo en portugués, delas condiciones de producción y los recuerdos de los pacientes en sanatorios en Europa dado a la ideología del régimen nazi, la interdicción de su decir y de los silenciamientos en producciones escritas en campos de concentración.

Al tratar el silencio para incluirlo en la perspectiva analítica del discurso, no se piensa en un silencio místico, ni en el silencio empírico, sino en el silencio que tiene su materialidad definida por la relación establecida entre decir y no decir.

Para fines de análisis, el corpus lo constituye una carta -entre cientos de ellasque circuló entre el matrimonio Ulli y Rudi, en la cual se buscar apuntar cómo los mecanismos de control del decir eran explícitamente aplicados, para percibir así la singularidad y regularidades del enunciado, los no-dichos y los silenciamientos presentes, de acuerdo con las condiciones de producción.

Se sabe que las condiciones de producción de un discurso constituyen un punto central para la $A D$, ya que para esa teoría toda actividad de lenguaje se da a partir de la colocación de los protagonistas del discurso en relación con el objeto discursivo. No se trata, pues, de la posición física de organismos humanos, sino de lugares determinados en la 
estructura de la formación social (dictador, paciente, exilado, etc.); la ocupación de determinada posición establece una relación del sujeto con una serie de formaciones imaginarias que involucra a los interlocutores y lo referente. Esa partir de estas relaciones que se producen los discursos.

\section{Aspectos teorico-metodológicos}

Las obras de Michel Foucault presentan una tesis general: "[...] 'la locura no es un hecho de la naturaleza', sino de la civilización [...]" (Sander, 2010, p. 382). La sociedad lleva ese preconcepto hasta la actualidad en la cual, incluso con los acontecimientos históricos recientes de la psiquiatría, las concepciones remotas de locura todavía están embebidas en la civilización actual. A partir de esa lectura también es perceptible que la locura es una producción social histórica mediada -en gran medida- por discursos, prácticas y producciones de representaciones sobre el estado de salud mental de los pacientes.

Para Foucault, las instituciones que nos gobiernan o que nos asisten, sean las dedicadas a la salud pública (hospitales, manicomios 0 asilos), al sistema penitenciario (casas de corrección, reformatorios, presidios y cadenas), de recuperación de adictos (clínicas especializadas), orfanatorios, seminarios, escuelas, universidades, centros técnicos, etc., no son más que "espacios de opresión" controlados por equipos dirigentes. El presente estudio tiene el objetivo de proponer discusiones al interpretar el proceso de producción y circulación de cartas entre novios en un sanatorio en la Alemania nazi. ¿Qué práctica discursiva se muestra en las cartas a partir de los mecanismos de interdicción del discurso?

La escuela, el orfanatorio, la universidad, el sanatorio, el hospital, la prisión y la cuadra pasaron a funcionar dentro de la misma dinámica con prácticamente los mismos regimientos internos que contenían objetivos de contención y domesticación de los seres humanos, aunque por diferentes vías. La vara, la palmatoria, la camisa de fuerza, el choque eléctrico, la medicación, el castigo corporal y los rigores de la instrucción militar forman una constelación de instrumentos opresivos que están a disposición de las corporaciones dominantes y sirven como afirmación de su poder en la tarea de sujeción de los individuos.

La prisión, junto con el manicomio, conformaron los espacios más extremistas y crueles de la opresión humana y sirvieron como modelo para las estructuras super autoritarias que surgieron a lo largo del siglo XX. A partir de esta constatación, Foucault (1979, p.45) enumeró entonces las instituciones que consideraba como intolerables: "los tribunales, los policías, los hospitales y asilos, la escuela, el servicio militar, la prensa, la televisión y el Estado.

Todas las técnicas 0 procedimientos efectuados en el asilo del siglo XIX-aislamiento, interrogatorio particular o público, tratamientoscastigos como la ducha, predicaciones morales, alentadores o reprensiones, disciplina rigurosa, trabajo obligatorio, recompensa, relaciones preferenciales entre el médico y algunos de sus enfermos, relaciones de vasallaje, de posesión, de domesticidad ya veces de servidumbre entre enfermo y médico- tenían por función hacer del personaje del médico el "maestro de la locura". Esto la hace manifestarse en su verdad cuando se esconde, cuando permanece sepultada y silenciosa, y en el que la domina, la calma y la absorbe después de haberla sabiamente desencadenado. (Foucault, 1979, p. 122).

El discurso que se pretende analizar en este artículo representa una importante fuente de investigación para el análisis del discurso 
(AD), pues, al transmitir factores ideológicos, históricos y sociales, acaba -aunque de forma implícita- por revelar las huellas de un discurso que muchas veces lleva a la exclusión de unos en detrimento de la ascensión de otros. A partir de las fuerzas ideológicas, impuestas socialmente por las clases dominantes que dictan qué hacer y cómo ser, las propagandas, de forma lingüística y visual, apelan a los conceptos y valores que gobiernan el comportamiento del hombre e influencian sus elecciones.

La analítica del poder de Foucault impone un desplazamiento en relación con el Estado en la medida en que identifica la existencia de una serie de relaciones de poder que se colocan fuera de él y que de ninguna manera se debe analizar en términos de soberanía, prohibición o imposición de una ley. Pero Foucault no quiere negar la importancia del Estado, su intención es demostrar que las relaciones de poder sobrepasan el nivel estatal y se extienden por toda la sociedad.

La historia de la locura, según Michel Foucault y su obra Historia de la locura (1972), presenta el dominio de la razón sobre la desrazón, al situar la razón como norma, y llevar la locura al exilio. Foucault trae a colación todo lo que se ha hecho contra la locura (exclusión, etc.). Es desde allí que es posible encontrar el camino hacia la razón, es decir que la razón se fortalece a costa de la desrazón.

Contrariamente a una historia tradicional de la psiquiatría que redirige a los (supuestos) orígenes de una locura inmemorial (griega, quizá egipcia), Foucault muestra una locura cocinada lentamente en el caldero de la historia occidental posterior al Renacimiento. Es así que se puede identificarla manera en que el horror, el temor y la admiración provocados por los locos en la época de la Stultifera Navis (Naus de los Insensatos) se transforman lentamente en la forma de escrutar la verdad del sujeto a través de la enfermedad mental en el siglo XIX.

Esto debido a que el establecimiento del hombre derramado que se llevó a cabo, sobre todo a partir del final del Renacimiento, no se hizo según un supuesto progreso natural de la raza humana, ni por medios de la aclaración y de la aceptación. No fue sin violencia y exclusión que la razón se estableció en el escenario occidental.

El régimen nazi fue, como afirma el filósofo-historiador francés Michel Foucault (1996), el desarrollo hasta el paroxismo de los nuevos mecanismos de poder que se habían introducido desde el siglo XVIII. Es el extremo de una política que toma el cuerpo como forma de manipulación de la población.

No hay ningún Estado más disciplinario, claro que el régimen nazi; tampoco hay Estado donde las regulaciones biológicas se adopten de una manera más densa y más insistente. El poder disciplinario, biopoder: todo ello recorrió, sostenía la sociedad nazi (asunción de lo biológico, de la procreación, de la herencia, asunción también de la enfermedad, de los accidentes). No hay sociedad a un tiempo más disciplinaria y más previsional que la que fue implantada, o en todo caso proyectada, por los nazis. El control de las eventualidades propias de los procesos biológicos era uno de los objetivos inmediatos del régimen. (Foucault, 19751976/2005, p. 309)

Algunas de estas cuestiones sobre la bio política y su consecuencia en la vida de las personas, a través de los procesos de control y sujeción por los que pasaron los individuos -en especial los judíos- eran los principales objetivos de esa política de control. 
Para Foucault, la bio-política se dirige, en suma, a los acontecimientos aleatorios que ocurren en una población considerada en su duración. De acuerdo con el filósofohistoriador, "se trata sobre todo de establecer mecanismos reguladores que, en esa población global con su campo aleatorio, van a poder fijar un equilibrio, mantener un promedio, establecer una especie de homeostasis, asegurar compensaciones" (Foucault, 1975-1976/2005, p. 293).

Foucault (1993) resalta que, si el genocidio es de hecho el sueño de los poderes modernos, no es por una vuelta al viejo derecho de matar. Es porque el poder se sitúa y se ejerce al nivel de la vida, de la especie, de la raza y de los fenómenos masivos de la población. Es lo que se puede observar al analizar el régimen nazi bajo el cual se mató legítimamente a aquellos que constituían una especie de peligro biológico para los demás, a ejemplo de los judíos, no arianos, homosexuales, gitanos, discapacitados mentales, entre otros.

Poblaciones enteras son llevadas a la destrucción mutua en nombre de la necesidad de vivir. Las masacres se volvieron vitales. Fue como gestores de la vida y de la supervivencia de los cuerpos y de la raza que tantos regímenes pudieron frenar tantas guerras, causando la muerte de tantos hombres. (Foucault, 1993, p. 149)

Por lo tanto, el poder pasó a establecer sobre la vida ya lo largo de todo su desarrollo sus puntos de fijación. Foucault afirma que a partir del siglo XVII y a través de dos formas principales se desarrolló1) el cuerpo como máquina (adiestramiento, utilidad, docilidad) y 2) el cuerpo como especie, cuerpo como mecánica del ser vivo y soporte de los procesos biológicos que.

Son asumidos mediante toda una serie de intervenciones y controles reguladores: una bio política de la población. Las disciplinas del cuerpo y las regulaciones de la población en torno a las cuales se desarrollaron la organización del poder sobre la vida. (Foucault, 1993, p. 152)

Con respecto a la noción de que el poder puede ser puramente represivo, Foucault comenta:

Cuando se definen los efectos del poder por la represión, se tiene una concepción puramente jurídica de este mismo poder; se identifica el poder a una ley que dice no. Lo fundamental sería la fuerza de la prohibición. Ahora bien, creo que esta es una noción negativa, estrecha y esquelética del poder que curiosamente todo el mundo ha aceptado. Si el poder fuese solo represivo, si no hiciera otra cosa que decir no, ¿crees que sería obedecido? Lo que hace que el poder se mantenga y que sea aceptado es simplemente que él no pesa s[o]lo como una fuerza que dice no, sino que de hecho él impregna, produce cosas, induce al placer, forma saber, produce discurso. Se debe considerarlo como una red productiva que atraviesa todo el cuerpo social mucho más que una instancia negativa que tiene por función reprimir. (Foucault, 1997, p. 8)

Al reflexionar sobre el corpus seleccionado, es decir las cartas producidas al interior del Sanatorio Sonnenfelds iqué discurso se podría producir a partir de la posición sujeto-paciente? ¿Qué fuerzas ideológicas establecerían aquello que podría y no podría ser dicho?

Por tratarse de documentos epistolares de la Segunda Guerra Mundial, estas producciones discursivas traen otro análisis de las condiciones de producción: el silencio como forma delo no-dicho.

Para Pêcheux, las condiciones se refieren a la posición del sujeto y posibilitan que un discurso se pronuncie o no. El lugar ocupado por este sujeto que anuncia interfiere en el sentido de su discurso, una vez que el sujeto 
está situado al interior de una relación de fuerzas: "un discurso es siempre pronunciado a partir de condiciones de producción dadas" (Pêcheux, 1987, p. 77).

Al hablar, el sujeto se vale de ciertos términos para construir su decir; sin embargo, podría decirlo de otra forma, con otras palabras. De ahí la razón para incluir en el análisis el concepto de silencio, puesto que cuando una formulación se materializa, otra se borra o silencia. Estas consideraciones nos hacen pensar en la cuestión del silencio político, tal como las trata Orlandi. Para la autora (2007, p. 81), el silencio político (o política del silencio) se puede presentar en forma de silencio constitutivo o en forma de silencio local (censura). En el silencio constitutivo el sujeto dice $X$ para no decir $Y$, es decir que al producir determinado enunciado, el sujeto silencia otros sentidos posibles, pero no deseados en aquel momento. La censura se caracteriza por la prohibición de la inserción del sujeto en determinadas formaciones discursivas, lo que afecta su identidad. En este sentido, Orlandi (1997, p. 81) dice que:

Es la interdicción manifiesta de la circulación del sujeto, por la decisión de un poder de palabra fuertemente regulado. En el autoritarismo, no hay reversibilidad posible en el discurso, es decir, el sujeto no puede ocupar diferentes posiciones: él sólo puede ocupar el "lugar" que le es destinado, para proponer los sentidos que no le son prohibidos. La censura afecta, de inmediato, a la identidad del sujeto.

Para Orlandi (2007), esa forma de lo no dicho es una vertiente cuyo origen está en el hecho de que el lenguaje es político y de que todo poder se acompaña de un silencio en su trabajo simbólico, una política del silencio que, para la autora, se da de dos maneras:

a. El silencio constitutivo, es decir, la parte del sentido que necesariamente se sacrifica, se apaga al decirse. Todo hablar silencia necesariamente. La actividad de nombrar es bien ilustrativa: toda denominación circunscribe el sentido del nombrado, rechaza así para el no-sentido todo lo que en él no está dicho;

b. El silencio local del tipo de censura y similares. Este silencio es el que se produce al prohibir algunos sentidos al circular, por ejemplo, en una forma de régimen político, en un grupo social determinado de una forma de sociedad específica, etc. (Orlandi, 2007, pp. 49-50)

Según la autora, el silencio no es visto solo en su negatividad, él significa, su no-decir contiene sentido, es fundador, por lo tanto, sostiene el principio de que el lenguaje es político. El silencio que pretendemos abordar en nuestros análisis es aquel que impregna las producciones escritas en los campos de concentración. Para Orlandi, como el discurso,

[...] sujeto y sentido se constituyen al mismo tiempo, al proceder de ese modo se prohíbe al sujeto ocupar ciertos "lugares", o mejor, se prohíben ciertas "posiciones" del sujeto. [...] La censura establece un juego de relaciones de fuerza por el cual se configura, de forma localizada, lo que, de lo diciente, no debe (no puede) ser dicho cuando el sujeto habla. (2007, pp. 76-77)

Se percibe aquí que la relación con el "decimario" es pues modificada cuando la censura interviene: no se trata más de lo decimario socio históricamente definido por las formaciones discursivas (el decir posible): no se puede decir lo que fue prohibido (decirlo debido). Es decir que no se puede decir lo que se puede decir.

Por lo tanto, es en esta perspectiva que, a través de la historia de la Segunda Guerra; de las memorias de los sanatorios; de la interdicción y censura de la palabra del sujeto paciente; de la ideología dominante de la época que se buscan las materialidades interpretativas 
necesarias para comprender que, para entrar en determinadas órdenes del discurso, el sujeto necesita estar inserto en un considerable número de reglas. Foucault ya resaltaba que,

[...] en toda sociedad la producción del discurso es a la vez controlada, seleccionada, organizada y redistribuida por cierto número de procedimientos que tienen por función conjurar sus poderes y peligros, dominar su acontecimiento aleatorio, esquivar su pesada y temible materialidad. (Foucault, 2018, pp. 8-9)

Con base en esta definición, se pasa ahora a analizar cómo la sociedad nazi interrumpió que los sujetos que estaban dentro o fuera de los centros de tratamientos psicológicos produjeran ciertos textos:

\section{Figura 3.}

\section{Carta casi incinerada por Dr. Karl Reichel}

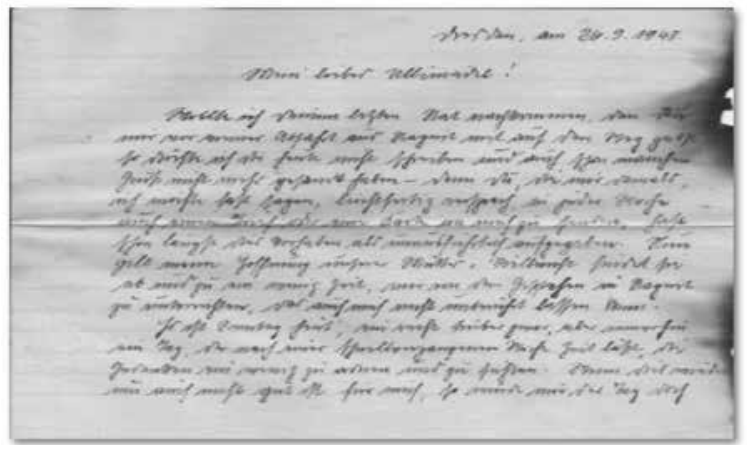

Fuente: Archivo personal del pesquisador Plinio Pereira

Figura 4.

Carta enviada a la paciente

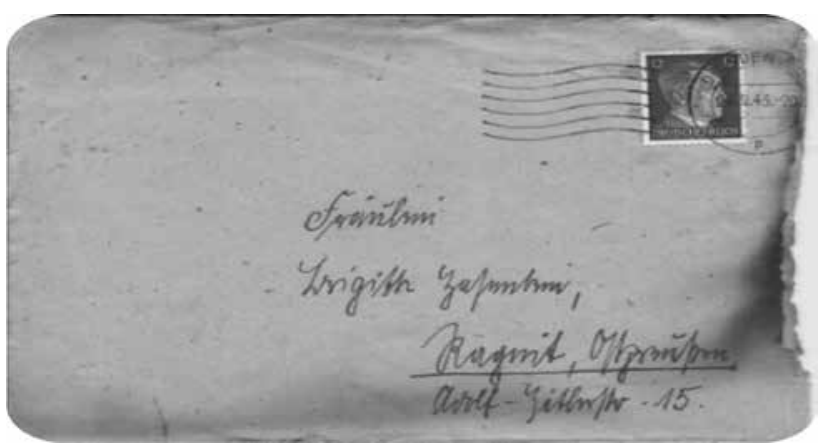

Fuente: Archivo personal del pesquisador Plinio Pereira

\section{Análisis}

El análisis se centra en una carta escrita entre una paciente del sanatorio Sonnenfelds y su novio. El caso de la figura 4 casi lo destruyo el médico Karl Reichel al final de la Segunda Guerra Mundial. Las cartas entre Ulli y Rudi son los últimos registros de ese sanatorio en el centro de Alemania.

\section{Figura 5.}

Transcripción de la escritura cursiva del documento para la mejor traducción del alemán

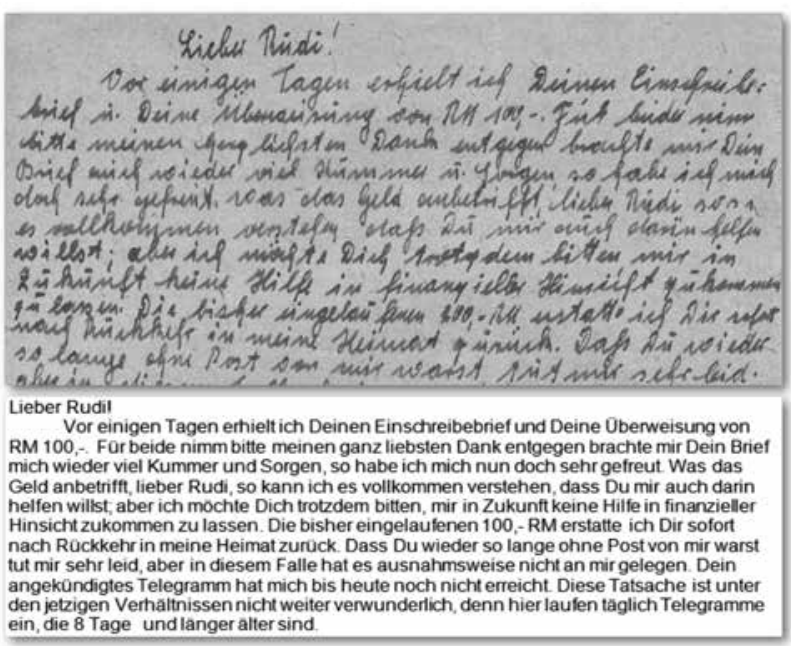

Fuente: Archivo personal del pesquisador Plinio Pereira

\section{Figura 6.}

Epístola al Rudi. Fragmento analizado (énfasis añadido en negrita)

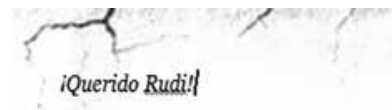

Recibi tu carta registrada hace unos días y tu envio de RM 100, - En lo que respecta al dinero, querido Rudi, puedo entender perfectamente que también quieras ayudarme con eso; pero quisiera pedirle, que no me envias más ninguna ayuda de carácter financiero. Te rindiré de los $R M 100$, - recibidos tan pronto como vuelva a mi tierra natal. Me siento que has quedado tanto tiempo sin recibir cartas mias, pero en este caso, excepcionalmente, la responsabilidad no es mía. Hasta hoy todavía no he recibido el telegrama que has ammciado.

Este hecho no es de extrañar frente a las actuales contingencias, pues aqui llegan todos los días telegramas fechados hace 8 días o más.

Mi salud va bien, excepto por la fiebre que volvió a manifestarse. Tube que restringir mis cestas ya que podrias harcelos afuera, queddndome solo le cyra del silencio,(Schweigekur)

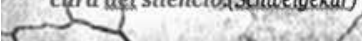

Fuente: Archivo personal del pesquisador Plinio Pereira 
La primera observación a señalar es que el intercambio de correspondencia solo sería posible si el remitente cumplía con las determinaciones de las reglas establecidas por la dirección del sanatorio. Es decir que el remitente tendría que satisfacer las exigencias. Sobre este hecho, Foucault afirma que "nadie entrará en el orden del discurso si no satisface ciertas exigencias o si no es, al principio, calificado para hacerlo". (Foucault, 2018, pp. 36-37). De hecho, los sujetos en condición de confinamiento no estaban autorizados a decir todo lo que sentían o pasaban dentro del sanatorio. Así como en los campos de concentración, en los sanatorios también había un control en la entrada de las cartas. En el fragmento Ulli dice que no había recibido su pedido.

De acuerdo con la ideología nazi "el poder está en todas partes; no porque englobe todo, sino porque proviene de todos los lugares “(Foucault, 1993, p. 89). Se percibe aquí que hay un embate entre poder y resistencia en lo que se refiere al decir que no es responsable por el no recibimiento de las cartas enviadas.

De forma acorde con el pensamiento de Foucault, las "estrategias de poder" se pueden definir como "un conjunto de los medios operados para hacer funcionar o para mantener un dispositivo de poder". (1995, p. 248). Pero evidencia también que "no hay una relación de poder sin resistencia, sin escapatoria o fuga, sin inversión eventual; toda relación de poder implica, entonces, al menos de modo virtual, una estrategia de lucha" (Foucault, 1995, p. 248). Foucault, expresa la importancia de las estrategias de confrontación dentro de las relaciones de poder y asegura que no hay una relación de poder sin resistencia.

Otros dos factores importantes para considerar en los mecanismos de control de las cartas son lo que "puede o no ser dicho" y el silencio en el acto de la censura. Este hecho se justifica en el pasaje "Hasta hoy no he recibido el telegrama que anunciaste". Se percibe aquí que hay un control en el funcionamiento del discurso del prisionero al obligarlo a no decir todo lo que sabe o siente. Sobre ello, afirma Foucault que "[...] se trata de determinar las condiciones de su funcionamiento, de imponer a los individuos que los pronuncian cierto número de reglas y así de no permitir que todo el mundo tenga acceso a ellos" (2018, pp. 36-37)

La expresión "no recibí" trae consigo el silenciamiento de no explicitar las condiciones reales de permanencia en el sanatorio. Así como atestigua Orlandi, en este enunciado se establece el.

[...] silencio local, una vez que se prohíbe que un sentido sea expuesto y que no permita que circule. Es el silencio fundador que produce un estado significativo para que el sujeto se inscrib[a] en el proceso de significación, incluso en la censura, haciendo significar por otros juegos de lenguaje, lo que le fue prohibido. La censura es un síntoma de que allí puede haber otro sentido. (Orlandi, 2007, p. 118)

Además de la censura en relación con la producción verbal, la fotografía tampoco podría servir como postal, ya que este denunciaría lo que en las palabras se silencia.

El silenciamiento producido por la censura lleva a un proceso de producción de sentidos silenciados. La expresión "yo estoy bien de salud" era visiblemente verbalizada en cartas de los campos de concentración, así como de los sanatorios. Esta expresión no traía solo una información vaga y silenciosa del estar del paciente o prisionero, sino una certeza de que está vivo. Es un proceso que trabaja la división entre el no decir y el decir, que impiden al sujeto ya la sociedad de trabajar en el movimiento de identidad y de elaborar históricamente los sentidos. El silencio interviene, por tanto, en la 
formación y en el movimiento de los sentidos y de ello deriva también el vínculo del no decir a la historia ya la ideología.

Por otro lado, la resistencia pasa a trabajar en la misma región de la censura, juega con las palabras del contexto hegemónico para resignificarlas. Por ejemplo, en el contexto de la Segunda Guerra Mundial, las fuentes bibliográficas y registros de memorias son las bases de sustentación y resistencia al régimen.

\section{Consideraciones "poco" finales}

Foucault concibe el poder en sus diversas dimensiones, es algo que está presente en todas las esferas sociales. En este trabajo se intentó comprender cómo se presentó el poder en el ámbito del régimen nazi de la Segunda Gran Guerra. Se evidencia así que la ideología impuesta no solo fue responsable de la segregación, separación, exclusión y exterminio de judíos, sino que también lo fue un control riguroso de los discursos. Este control se hacía mediante el establecimiento

\section{Referencias}

Arendt, H. (1989). Los límites del Totalitarismo (R. Raposo, trad.). Compañía de las Letras.

Foucault. M. (1970/2008). El orden del discurso: clase inaugural en el Collège de France, pronunciada el 2 de diciembre de 1970(L. Fraga de Almeida Sampaio, trd.). (20ㅇe ed.) Ediciones Loyola.

Foucault. M. (1975-1976/2005). En defensa de la sociedad. Curso enel College de France (19751976) (M. E. Galvão, trad.). Martins Pontes.

Foucault. M. (1996). Genealogía del racismo. La Plata: Altamira.

Foucault. M. (1972). História da e la locura. São Paulo: Perspectiva, 1972. de reglas de conducta que evaluaban lo que podía o no decirse a través del intercambio de correspondencia entre pacientes del Sanatorio Sonnenfelds.

A través del recorrido histórico relativo a las manifestaciones del poder, del régimen de control y prohibición, se percibe en el análisis del fragmento dela carta producidas en el sanatorio que a los sujetos internados se lessometía a decir, no lo que querían, sino a producir discursos que silenciaban las verdaderas las condiciones por las que pasaban todos los días. Esta carta, aparentemente quemada por el médico y propietario, Karl Reichel revela que hay mucho más por investigar y desmitificar en lo que se refiere a las producciones epistolares de la Segunda Guerra Mundial. El primer paso dado, aunque inicial, fue comprender cómo dentro del Sanatorio Sonnenfelds, el poder de un aparato de Estado se controlaba en el discurso y su no cumplimiento acarreaba la interdicción discursiva de los documentos.

Foucault. M. (1979). Microfísica del Poder (R. Machado, trad.) (18ํed.). Edições Graal,

Foucault. M. (1993). La historia de la sexualidad I: la voluntad de saber (Maria Thereza da Costa Albuquerque y J. A. Guilhon Albuquerque, trads.).Graal.

Foucault. M. (1995). El Sujeto y el Poder. EnP. Rabinow y H. Dreyfus, Michel Foucault. Una trayectoria filosófica (pp. 231-249). Forense Universitária.

Foucault. M. (2018). El nacimiento de la Clínica (R. Machado, trad.) ( $7^{-0}$ ed.). Florense Universitária.

Le Goff, J. (1999). Memoria. En Historia y Memoria (B. Leitão et al., trad) (pp. 423-483). Editora da Unicamp. 
Orlandi, E. P. (2002). Análisis del discurso: principios y procedimientos ( $4^{\circ}$ ed.). Puentes.

Orlandi, E. P. (2007). Las formas del silencio: en el movimiento de los sentidos ( $4^{\circ}$ ed.). Unicamp.

Orlandi, E. P (1997). Gestos de leitura: da História no Discurso. Campinas: UNICAMP.

Pêcheux, M. (1987). El discurso: estructura o acontecimiento (E. Orlandi, trad.). Pontes Editora.

Pêcheux, M. (1988). Semántica y discurso: una crítica a la afirmación de lo obvio (E. Orlandi et al., trad.). Unicamp.
Pêcheux, M. (1999). Papel de la memoria. En P. Achard, Papel de la memoria (J. Horta Nunes, trad.) Pontes.

Sander, J. (2010). La caja de herramientas de Michel Foucault, la reforma psiquiátrica y los desafíos contemporáneos. Psicología y Sociedad, 22(2), 382-387.

Sülzhayn, Reinhard GlaB, http://www.glassportal.privat.tonline.de/suelzhayn/ architekt/mohr_und_weidner1.2.htm WACHENFELD, Leonhard, 2017, p. 01

Wiener, A. (2007). 64735 From A Name to A Number: La Holocaust Survivor de Autobiografía. Author House. 